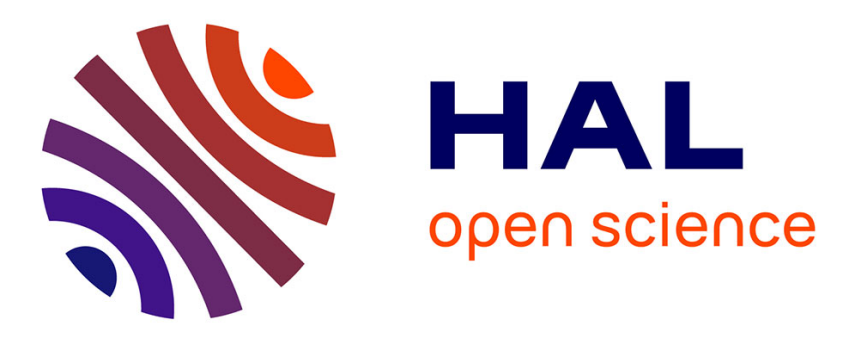

\title{
Migration of imaginary roots of multiplicity three and four under small deviation of two delays in time-delay systems
}

\author{
Dina Irofti, Keqin Gu, Islam Boussaada, Silviu-Iulian Niculescu
}

\section{To cite this version:}

Dina Irofti, Keqin Gu, Islam Boussaada, Silviu-Iulian Niculescu. Migration of imaginary roots of multiplicity three and four under small deviation of two delays in time-delay systems. 15th European Control Conference (ECC 2016), Jun 2016, Aalborg, Denmark. 10.1109/ecc.2016.7810535 . hal01418212

\author{
HAL Id: hal-01418212 \\ https://hal.science/hal-01418212
}

Submitted on 6 Jan 2017

HAL is a multi-disciplinary open access archive for the deposit and dissemination of scientific research documents, whether they are published or not. The documents may come from teaching and research institutions in France or abroad, or from public or private research centers.
L'archive ouverte pluridisciplinaire HAL, est destinée au dépôt et à la diffusion de documents scientifiques de niveau recherche, publiés ou non, émanant des établissements d'enseignement et de recherche français ou étrangers, des laboratoires publics ou privés. 


\title{
Migration of imaginary roots of multiplicity three and four under small deviation of two delays in time-delay systems
}

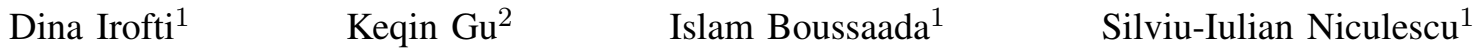

\begin{abstract}
This paper studies the migration pattern of characteristic imaginary roots of multiplicity three and four in time-delay systems with two delays when the delay parameters undergo small deviations. Stability analysis for such problems is often based on Puiseux series, as multiple roots are not differentiable with respect to delay parameters. However, in this paper the approach is more traditional without using Puiseux series. In the case of triple roots, we show that the stability crossing curves are smooth; when a perturbation occurs in the delay parameter space, two roots move to one half-plane and one root to the other half-plane. The case of quadruple root is more complicated as the stability crossing curve has a cusp. Thus, in the neighbourhood of the critical point, the delay parameter space is divided in an $\mathrm{S}$-sector and a $\mathrm{G}$-sector. When the parameters move into the G-sector, two roots move to the right half-plane, and the other two roots move to the left half-plane. When the parameters move into the S-sector, then three of the roots move to one half-plane, and the remaining root moves to the other half-plane, depending on the conditions.
\end{abstract}

\section{INTRODUCTION}

As many dynamic process contain some aftereffect (delay) phenomenon, the scientific community has a great interest in time-delay systems. However, the stability analysis of such systems is not an easy task as they belong to the class of functional differential equations. A very useful method for such an analysis is D-decomposition method [1]. Suppose the system depends on some parameters, the idea of D-decomposition method is to find the values of these parameters at which the number of the characteristic roots in the right half-plane changes. Such values divide the parameter space into regions. The method is especially valuable for analyzing time-delay systems [2] [3] [5]. When the parameters are the delays, this method is also known as $\tau$-decomposition method [4] [5].

In this paper we consider a case not sufficiently discussed in the literature (see for instance [6], [7]), namely when the system's characteristic equation has multiple imaginary roots for some parameters. The stability analysis of systems with two delays and without multiple imaginary roots is discussed in [8]. Next, [9] presents an analysis for the case of double imaginary roots, it was shown that the local stability crossing curve has a cusp as shown in Figure 1, and an explicit criterion is given regarding how the double characteristic

\footnotetext{
${ }^{1}$ Dina Irofti, Islam Boussaada, and Silviu-Iulian Niculescu are Laboratoire des Signaux et Systèmes (L2S, UMR CNRS 8506), CNRSCentraleSupelec-University Paris-Sud, 3, rue Joliot Curie, 91190, Gifsur-Yvette, France. Dina.Irofti, Islam.Boussaada, Silviu.Niculescull2s.centralesupelec.fr

${ }^{2} \mathrm{Keqin} \mathrm{Gu}$ is with Department of Mechanical and Industrial Engineering, Southern Illinois University Edwardsville, Edwardsville, Illinois 62026, USA. kguesiue.edu
}

imaginary roots migrate as the delay parameters deviate from the critical values.

The cases of roots with multiplicity three and four have recently come into attention of the control community, and some work has been already done in connection with inverted pendulum (see, for instance, the bifurcation analysis of triplezero eigenvalue in [10] and [11]).

Puiseux series are often used in the literature for the stability analysis in the case of multiple roots (see [12] and Part II, Chapter 5 of [13]). This approach is also important for time-delay systems (see for instance [14], [15], [7], where stability analysis is based on Puiseux series). We also mention [16], where delay blocks have been used in order to control a chain of oscillators.

In this paper, we study the case of imaginary characteristic roots of multiplicity three and four, and show that the stability analysis can be based on a more conventional approach without using Puiseux series. We shall see that if the system has an imaginary root of multiplicity four, then the stability crossing curve has a cusp in the parameter space. This is not the case for triple roots. The paper is structured as follows: Section II states the problem and introduces the notation. In Sections III and IV we give the results concerning the behaviours of characteristic roots when a small change in the parameters occurs, for the case of triple and quadruple roots, respectively. The last section contains some concluding remarks.

\section{PROBLEM FORMULATION}

Consider a system with two delays, $\tau_{1}$ and $\tau_{2}$, with the characteristic equation

$$
p\left(s, \tau_{1}, \tau_{2}\right)=p_{0}(s)+p_{1}(s) e^{-\tau_{1} s}+p_{2}(s) e^{-\tau_{2} s}=0,
$$

where $p_{k}(s), k=0,1,2$ are polynomials of $s$ with real coefficients, $\tau_{1}, \tau_{2}$ are independent positive delays, and $s$ is the Laplace variable. For $\tau_{1}=\tau_{10}, \tau_{2}=\tau_{20}$, we assume $p\left(s, \tau_{1}, \tau_{2}\right)$ has an imaginary root $s_{0}=i \omega_{0}$ of $m^{t h}$ order. In other words,

$$
\begin{aligned}
& \left.\frac{\partial^{k} p}{\partial s^{k}}\right|_{\substack{s=s_{0} \\
\tau_{1}=\tau_{10} \\
\tau_{2}=\tau_{20}}}=0, \text { for } k=0 \ldots m-1 \\
& \left.\frac{\partial^{m} p}{\partial s^{m}}\right|_{\substack{s=s_{0} \\
\tau_{1}=\tau_{10} \\
\tau_{2}=\tau_{20}}} \neq 0 .
\end{aligned}
$$

The case of $m=2$ (double roots) is presented in [9]. This paper studies the case of $m=3$ (triple roots) and $m=4$ (quadruple roots). 
Throughout this paper, we make the following "least degeneracy" assumption:

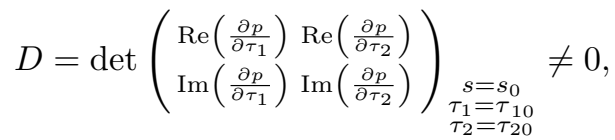

where $\operatorname{Re}(\cdot)$ denotes the real part, and $\operatorname{Im}(\cdot)$ denotes the imaginary part of a complex number. In view of implicit function theorem, a consequence of the assumption (4) is that the characteristic equation (1) defines the pair $\left(\begin{array}{ll}\tau_{1} & \tau_{2}\end{array}\right)$ in a small neighbourhood of the critical point $\left(\tau_{10} \tau_{20}\right)$ as a function of $s$ in a sufficiently small neighbourhood of $s_{0}$.

Introduce the notation

$$
\mathcal{N}_{\varepsilon}\left(x_{0}\right)=\left\{x|| x-x_{0} \mid<\varepsilon\right\} .
$$

Then, in a sufficiently small neighbourhood $\mathcal{N}_{\delta}\left(s_{0}\right)$ of $s_{0}$, we can define (see proposition 1 in [9]) two functions, $\tau_{1}(s)$ and $\tau_{2}(s)$, differentiable up to an arbitrary order, as the unique solution of characteristic equation (1) in a small neighbourhood, $\left(\tau_{1}(s) \tau_{2}(s)\right) \in \mathcal{N}_{\varepsilon}\left(\tau_{10}, \tau_{20}\right)$ (but this characteristic equation may have other solutions outside the of $\left.\mathcal{N}_{\varepsilon}\left(\tau_{10}, \tau_{20}\right)\right)$.

Define local stability crossing curve as the set

$$
\begin{aligned}
& \mathcal{T}_{\left(\omega_{0}, \tau_{10}, \tau_{20}\right)}= \\
& \quad\left\{\left(\tau_{1}(i \omega), \tau_{2}(i \omega)\right) \in \mathcal{N}_{\varepsilon}\left(\tau_{10}, \tau_{20}\right) \mid i \omega \in \mathcal{N}_{\delta}\left(i \omega_{0}\right)\right\} .
\end{aligned}
$$

This curve divides $\mathcal{N}_{\varepsilon}\left(\tau_{10}, \tau_{20}\right)$ into two regions. We will study how the triple or quadruple roots migrate as the delay parameters $\left(\tau_{1} \tau_{2}\right)$ move into one of these two regions.

For the sake of convenience, we also define the positive local stability crossing curve as

$$
\begin{aligned}
& \mathcal{T}_{\left(\omega_{0}, \tau_{10}, \tau_{20}\right)}^{+}= \\
& \left\{\left(\tau_{1}(i \omega), \tau_{2}(i \omega)\right) \in \mathcal{N}_{\varepsilon}\left(\tau_{10}, \tau_{20}\right) \mid i \omega \in \mathcal{N}_{\delta}\left(i \omega_{0}\right), \omega>\omega_{0}\right\},
\end{aligned}
$$

and the negative local stability crossing curve as

$$
\begin{aligned}
& \mathcal{T}_{\left(\omega_{0}, \tau_{10}, \tau_{20}\right)}^{-}= \\
& \left\{\left(\tau_{1}(i \omega), \tau_{2}(i \omega)\right) \in \mathcal{N}_{\varepsilon}\left(\tau_{10}, \tau_{20}\right) \mid i \omega \in \mathcal{N}_{\delta}\left(i \omega_{0}\right), \omega<\omega_{0}\right\} .
\end{aligned}
$$

\section{MULTIPLICITY THREE}

In this section, we study the migration of triple roots.

Theorem 1: Suppose system (1) satisfies (2) and (3) for $m=3$, and assumption (4) holds. Then, as $\left(\tau_{1} \tau_{2}\right)$ moves from $\left(\begin{array}{ll}\tau_{10} & \tau_{20}\end{array}\right)$ to one of the two regions of $\mathcal{N}_{\varepsilon}\left(\tau_{10}, \tau_{20}\right)$ divided up by $\mathcal{T}_{\left(\omega_{0}, \tau_{10}, \tau_{20}\right)}$, at least one root moves to the right half-plane, and one other root moves to the left half-plane. The remaining root may move to either the left half-plane, or the right half-plane. Specifically:

Case i. $D>0$ and $\left(\begin{array}{ll}\tau_{1} & \tau_{2}\end{array}\right)$ moves in the region on the clockwise side of $\mathcal{T}_{\left(\omega_{0}, \tau_{10}, \tau_{20}\right)}^{+}$and on the counterclockwise side of $\mathcal{T}_{\left(\omega_{0}, \tau_{10}, \tau_{20}\right)}^{-}$. In this case, two characteristic roots of (1) move to the right-half complex plane, and the third root moves to the left-half plane.

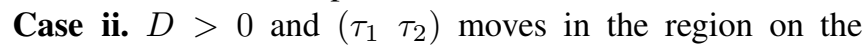
clockwise side of $\mathcal{T}_{\left(\omega_{0}, \tau_{10}, \tau_{20}\right)}^{-}$and on the counterclockwise side of $\mathcal{T}_{\left(\omega_{0}, \tau_{10}, \tau_{20}\right)}^{+}$. In this case, two characteristic roots of
(1) move to the left-half complex plane, and the third root moves to the right-half plane.

Case iii. $D<0$ and $\left(\begin{array}{ll}\tau_{1} & \tau_{2}\end{array}\right)$ moves in the region on the clockwise side of $\mathcal{T}_{\left(\omega_{0}, \tau_{10}, \tau_{20}\right)}^{-}$and on the counterclockwise side of $\mathcal{T}_{\left(\omega_{0}, \tau_{10}, \tau_{20}\right)}^{+}$. In this case, two characteristic roots of (1) move to the right-half complex plane, and the third root moves to the left-half plane.

Case iv. $D<0$ and $\left(\tau_{1} \tau_{2}\right)$ moves in the region on the clockwise side of $\mathcal{T}_{\left(\omega_{0}, \tau_{10}, \tau_{20}\right)}^{+}$and on the counterclockwise side of $\mathcal{T}_{\left(\omega_{0}, \tau_{10}, \tau_{20}\right)}^{-}$. In this case, two characteristic roots of (1) move to the left-half complex plane, and the third root moves to the right-half plane.

Proof: In the complex plane consider a point $s$ in the neighbourhood of $s_{0}$, let

$$
s=s_{0}+u e^{i \theta}
$$

Denote

$$
\gamma=e^{i \theta}=\frac{\partial s}{\partial u}
$$

Differentiate (1) with respect to $u$ with the angular variable $\theta$ fixed (equivalently with $\gamma$ fixed), and consider $\tau_{1}(s)$ and $\tau_{2}(s)$ as functions of $u$ and $\theta$. This yields:

$$
\frac{\partial p}{\partial \tau_{1}} \frac{\partial \tau_{1}}{\partial u}+\frac{\partial p}{\partial \tau_{2}} \frac{\partial \tau_{2}}{\partial u}+\frac{\partial p}{\partial s} \gamma=0
$$

Setting $u=0$ and using equation (2) for $k=1$ in (6), we obtain

$$
\left(\begin{array}{ll}
\operatorname{Re}\left(\frac{\partial p}{\partial \tau_{1}}\right) & \operatorname{Re}\left(\frac{\partial p}{\partial \tau_{2}}\right) \\
\operatorname{Im}\left(\frac{\partial p}{\partial \tau_{1}}\right) & \operatorname{Im}\left(\frac{\partial p}{\partial \tau_{2}}\right)
\end{array}\right) \underbrace{}_{\substack{s=s_{0} \\
\tau_{1}=\tau_{10} \\
\tau_{2}=\tau_{20}}}\left(\begin{array}{l}
\frac{\partial \tau_{1}}{\partial u} \\
\frac{\partial \tau_{2}}{\partial u}
\end{array}\right)_{u=0}=0,
$$

from which we conclude

$$
\left(\begin{array}{l}
\frac{\partial \tau_{1}}{\partial u} \\
\frac{\partial \tau_{2}}{\partial u}
\end{array}\right)_{u=0}=0
$$

in view of (4) and (5).

Differentiating (6) with respect to $u$ again yields

$$
\begin{array}{r}
\frac{\partial^{2} p}{\partial \tau_{1}^{2}}\left(\frac{\partial \tau_{1}}{\partial u}\right)^{2}+2 \frac{\partial^{2} p}{\partial \tau_{1} \partial \tau_{2}} \frac{\partial \tau_{1}}{\partial u} \frac{\partial \tau_{2}}{\partial u}+2 \frac{\partial^{2} p}{\partial \tau_{1} \partial s} \frac{\partial \tau_{1}}{\partial u} \gamma+ \\
+\frac{\partial p}{\partial \tau_{1}} \frac{\partial^{2} \tau_{1}}{\partial u^{2}}+\frac{\partial^{2} p}{\partial \tau_{2}^{2}}\left(\frac{\partial \tau_{2}}{\partial u}\right)^{2}+2 \frac{\partial^{2} p}{\partial \tau_{2} \partial s} \frac{\partial \tau_{2}}{\partial u} \gamma+ \\
+\frac{\partial p}{\partial \tau_{2}} \frac{\partial^{2} \tau_{2}}{\partial u^{2}}+\frac{\partial^{2} p}{\partial s^{2}} \gamma^{2}=0
\end{array}
$$

Similar to the way we obtained (7) from (6), we may conclude from (8) using (2) for $k=2$ and equation (7) that

$$
\left(\begin{array}{c}
\frac{\partial^{2} \tau_{1}}{\partial u^{2}} \\
\frac{\partial^{2} \tau_{2}}{\partial u^{2}}
\end{array}\right)_{u=0}=0
$$


Differentiating (8) again with respect to $u$ yields

$$
\begin{array}{r}
\frac{\partial^{3} p}{\partial \tau_{1}^{3}}\left(\frac{\partial \tau_{1}}{\partial u}\right)^{3}+3 \frac{\partial^{2} p}{\partial \tau_{1}^{2}} \frac{\partial^{2} \tau_{1}}{\partial u^{2}} \frac{\partial \tau_{1}}{\partial u}+ \\
+3 \frac{\partial^{3} p}{\partial \tau_{1}^{2} \partial \tau_{2}}\left(\frac{\partial \tau_{1}}{\partial u}\right)^{2} \frac{\partial \tau_{2}}{\partial u}+3 \frac{\partial^{3} p}{\partial \tau_{1}^{2} \partial s}\left(\frac{\partial \tau_{1}}{\partial u}\right)^{2} \gamma+ \\
+3 \frac{\partial^{2} p}{\partial \tau_{1} \partial \tau_{2}} \frac{\partial^{2} \tau_{1}}{\partial u^{2}} \frac{\partial \tau_{2}}{\partial u}+3 \frac{\partial^{3} p}{\partial \tau_{1} \partial \tau_{2}^{2}} \frac{\partial \tau_{1}}{\partial u}\left(\frac{\partial \tau_{2}}{\partial u}\right)^{2}+ \\
+3 \frac{\partial^{2} p}{\partial \tau_{1} \partial \tau_{2}} \frac{\partial \tau_{1}}{\partial u} \frac{\partial^{2} \tau_{2}}{\partial u^{2}}+6 \frac{\partial^{3} p}{\partial \tau_{1} \partial \tau_{2} \partial s} \frac{\partial \tau_{1}}{\partial u} \frac{\partial \tau_{2}}{\partial u} \gamma+ \\
+3 \frac{\partial^{3} p}{\partial \tau_{1} \partial s^{2}} \frac{\partial \tau_{1}}{\partial u} \gamma^{2}+\frac{\partial p}{\partial \tau_{1}} \frac{\partial^{3} \tau_{1}}{\partial u^{3}}+3 \frac{\partial^{2} p}{\partial \tau_{1} \partial s} \frac{\partial^{2} \tau_{1}}{\partial u^{2}} \gamma+ \\
+\frac{\partial^{3} p}{\partial \tau_{2}^{3}}\left(\frac{\partial \tau_{2}}{\partial u}\right)^{3}+3 \frac{\partial^{2} p}{\partial \tau_{2}^{2}} \frac{\partial \tau_{2}}{\partial u} \frac{\partial^{2} \tau_{2}}{\partial u^{2}}+ \\
+3 \frac{\partial^{3} p}{\partial \tau_{2}^{2} \partial s}\left(\frac{\partial \tau_{2}}{\partial u}\right)^{2} \gamma+3 \frac{\partial^{2} p}{\partial \tau_{2} \partial s} \frac{\partial^{2} \tau_{2}}{\partial u^{2}} \gamma+ \\
+3 \frac{\partial^{3} p}{\partial \tau_{2} \partial s^{2}} \frac{\partial \tau_{2}}{\partial u} \gamma^{2}+\frac{\partial p}{\partial \tau_{2}} \frac{\partial^{3} \tau_{2}}{\partial u^{3}}+\frac{\partial^{3} p}{\partial s^{3}} \gamma^{3}=0
\end{array}
$$

If we set $u=0$ and use (7) and (9) in equation (10), we obtain

$$
\left.\left(\frac{\partial p}{\partial \tau_{1}} \frac{\partial^{3} \tau_{1}}{\partial u^{3}}+\frac{\partial p}{\partial \tau_{2}} \frac{\partial^{3} \tau_{2}}{\partial u^{3}}+\frac{\partial^{3} p}{\partial s^{3}} \gamma^{3}\right)\right|_{\substack{s=s_{0} \\ \tau_{1}=\tau_{10} \\ \tau_{2}=\tau_{20}}}=0
$$

or

$$
\left.\left(\frac{\partial p}{\partial \tau_{1}} \frac{\partial^{3} \tau_{1}}{\partial u^{3}}+\frac{\partial p}{\partial \tau_{2}} \frac{\partial^{3} \tau_{2}}{\partial u^{3}}\right)\right|_{\substack{s=s_{0} \\ \tau_{1}=\tau_{10} \\ \tau_{2}=\tau_{20}}}=\left.\left(-\frac{\partial^{3} p}{\partial s^{3}} \gamma^{3}\right)\right|_{\substack{s=s_{0} \\ \tau_{1}=\tau_{10} \\ \tau_{2}=\tau_{20}}}
$$

We separate real and imaginary part to obtain

$$
\begin{aligned}
& \left(\begin{array}{cc}
\operatorname{Re}\left(\frac{\partial p}{\partial \tau_{1}}\right) & \operatorname{Re}\left(\frac{\partial p}{\partial \tau_{2}}\right) \\
\operatorname{Im}\left(\frac{\partial p}{\partial \tau_{1}}\right) & \operatorname{Im}\left(\frac{\partial p}{\partial \tau_{2}}\right)
\end{array}\right) \underbrace{}_{\substack{s=s_{0} \\
\tau_{1}=\tau_{10} \\
\tau_{2}=\tau_{20}}}\left(\begin{array}{c}
\frac{\partial^{3} \tau_{1}}{\partial u^{3}} \\
\frac{\partial^{3} \tau_{2}}{\partial u^{3}}
\end{array}\right)_{\substack{s=s_{0} \\
\tau_{1}=\tau_{10} \\
\tau_{2}=\tau_{20}}}
\end{aligned}
$$

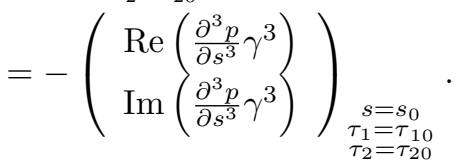

Thus

$$
\begin{aligned}
& \left(\begin{array}{c}
\frac{\partial^{3} \tau_{1}}{\partial u^{3}} \\
\frac{\partial^{3} \tau_{2}}{\partial u^{3}}
\end{array}\right)_{\substack{s=s_{0} \\
\tau_{1}=\tau_{10} \\
\tau_{2}=\tau_{20}}}= \\
& -\left(\operatorname{Re}\left(\frac{\partial p}{\partial \tau_{1}}\right) \operatorname{Re}\left(\frac{\partial p}{\partial \tau_{2}}\right)\right)^{-1}\left(\operatorname{Re}\left(\frac{\partial^{3} p}{\partial s^{3}} \gamma^{3}\right)\right) \\
& \left.\operatorname{Im}\left(\frac{\partial p}{\partial \tau_{1}}\right) \operatorname{Im}\left(\frac{\partial p}{\partial \tau_{2}}\right)\right) \begin{array}{l}
\partial^{3}=s_{0} \\
\tau_{1}=\tau_{10} \\
\tau_{2}=\tau_{20}
\end{array}
\end{aligned}
$$

Using Lemma 6 in [9] and in view of (11), we know that a $90^{\circ}$ counterclockwise rotation of $\gamma$ in the complex plane will generate a $270^{\circ}$ rotation in $\tau_{1}-\tau_{2}$ parameter space, in the counterclockwise direction if $D>0$, and in the clockwise direction if $D<0$.

Accounting for higher order terms, the situation is illustrated in Figure 2 for Cases $\mathrm{i}$ and ii $(D>0)$, and in Figure

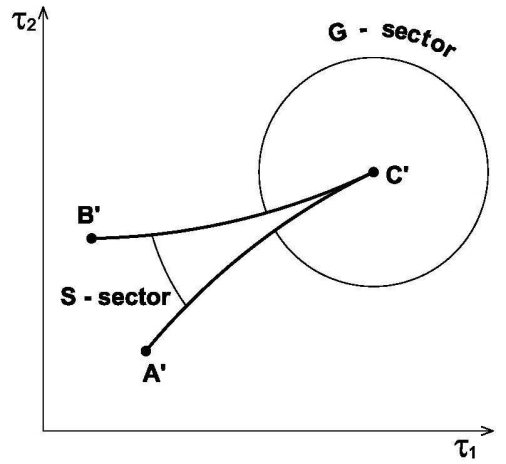

Fig. 1. G-sector and S-sector.

3 for Cases iii and iv $(D<0)$. In both Figures 2 and 3, the line segment $C D$ in the diagram on the left is mapped to $C^{\prime} D^{\prime}$ (in $\left.\operatorname{Re}(+)\right)$ in the diagram on the right. Similarly, $C B$, $C E$ and $C A$ in the diagram on the left are mapped to $C^{\prime} B^{\prime}$ (in $\operatorname{Im}(+)$ or $\mathcal{T}_{\left(\omega_{0}, \tau_{10}, \tau_{20}\right)}^{+}$), $C^{\prime} E^{\prime}$ (in $\operatorname{Re}(-)$ ) and $C^{\prime} A^{\prime}$ (in $\operatorname{Im}(-)$ or $\left.\mathcal{T}_{\left(\omega_{0}, \tau_{10}, \tau_{20}\right)}^{-}\right)$in the diagram on the right.

Consider Cases $i$ and ii shown in Figure 2. The arc $B D$ in darker solid curve on the diagram on the left is mapped to the arc $B^{\prime} D^{\prime}$ in the same line type on the diagram on the right that goes around point $C^{\prime}$ about $270^{\circ}$. Therefore, region $I$ bounded by $B C, C D$, and arc $D B$ in the diagram on the left is mapped bijectively to the singly connected region bounded by the arcs $B^{\prime} C^{\prime}, C^{\prime} D^{\prime}$ and the darker solid arc $D^{\prime} B^{\prime}$, which we will denote as $I^{\prime}$, in the diagram on the right. Similarly, region $I I$ is mapped bijectively to region $I I^{\prime}$ bounded by $E^{\prime} C^{\prime}, C^{\prime} B^{\prime}$ and the darker dotted arc $B^{\prime} E^{\prime}$, region $I I I$ is mapped bijectively by region $I I I^{\prime}$ bounded by $A^{\prime} C^{\prime}$, $C^{\prime} E^{\prime}$ and the ligher solid arc $E^{\prime} A^{\prime}$, region $I V$ is mapped bijectively to region $I V^{\prime}$ bounded by $D^{\prime} C^{\prime}, C^{\prime} A^{\prime}$ and the ligher dotted arc $A^{\prime} D^{\prime}$. Notice, the region on the clockwise side of $\operatorname{Im}(+)$ (or $\left.\mathcal{T}_{\left(\omega_{0}, \tau_{10}, \tau_{20}\right)}^{+}\right)$and on the counterclockwise side of $\operatorname{Im}(-)$ ( or $\left.\mathcal{T}_{\left(\omega_{0}, \tau_{10}, \tau_{20}\right)}^{-}\right)$in the neighbourhood of $C^{\prime}$ (or $\left(\tau_{10}, \tau_{20}\right)$ ) may be expressed as $I^{\prime} \cap\left(I I^{\prime} \cup I I I^{\prime}\right) \cap I V^{\prime}$. Therefore, for any $\left(\tau_{1}, \tau_{2}\right)$ in this region, there must be one root in region $I$, one root in either region $I I$ or region $I I I$, and one root in region $I V$. In other words, there must be two roots on the right half plane, and one root on the left half plane. This proves Case i. Case ii can be shown by noticing that the region on the clockwise side of $\operatorname{Im}(-)$ (or $\mathcal{T}_{\left(\omega_{0}, \tau_{10}, \tau_{20}\right)}^{-}$) and on the counterclockwise side of $\operatorname{Im}(+)$ (or $\left.\mathcal{T}_{\left(\omega_{0}, \tau_{10}, \tau_{20}\right)}^{+}\right)$in the neighborhood of $\left(\tau_{10}, \tau_{20}\right)$ may be expressed as $\left(I^{\prime} \cup I V^{\prime}\right) \cap I I^{\prime} \cap I I I^{\prime}$. Cases iii and iv may be shown similarly.

Remark 1: Note that

$$
\left(\frac{\partial^{3} \tau_{1}}{\partial u^{3}}\right)_{\gamma=-i}=-\left(\frac{\partial^{3} \tau_{1}}{\partial u^{3}}\right)_{\gamma=i}
$$

in view of (11). This means, in view of (7) and (9), that $\mathcal{T}_{\left(\omega_{0}, \tau_{10}, \tau_{20}\right)}^{+}$has the same tangent as $\mathcal{T}_{\left(\omega_{0}, \tau_{10}, \tau_{20}\right)}^{-}$at $\left(\tau_{10} \tau_{20}\right)$. Thus, $\mathcal{T}_{\left(\omega_{0}, \tau_{10}, \tau_{20}\right)}$ is a smooth curve. In other words, unlike the double root case discussed in [9], the stability crossing 

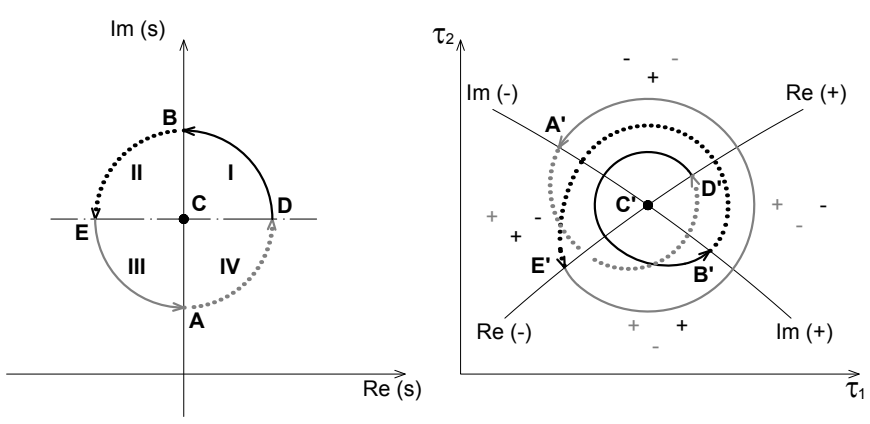

Fig. 2. The mapping $\left(\tau_{1}(s), \tau_{2}(s)\right)$ in a neighborhood of $s_{0} . D>0$.

curve is smooth without a cusp at $\left(\begin{array}{ll}\tau_{10} & \tau_{20}\end{array}\right)$.

\section{MULTIPLICITY FOUR}

In this section we study the migration of quadruple roots. For system (1), $s_{0}$ is a quadruple root if conditions (2) and (3) hold for $m=4$.

Parameterize $s$ by $u$ and $\theta$ (or $\gamma$ ) as in (5). From (7), (9) and (11), we immediately conclude

$$
\left(\begin{array}{l}
\frac{\partial^{k} \tau_{1}}{\partial u^{k}} \\
\frac{\partial^{k} \tau_{2}}{\partial u^{k}}
\end{array}\right)_{u=0}=0 \quad \text { for } k=1,2,3
$$

The above is true for $k=3$ due to (11) and equation (2) for $k=3$.

Differentiate (10) again with respect to $u$, taking into account (12); we obtain

$$
\left(\frac{\partial p}{\partial \tau_{1}} \frac{\partial^{4} \tau_{1}}{\partial u^{4}}+\frac{\partial p}{\partial \tau_{2}} \frac{\partial^{4} \tau_{2}}{\partial u^{4}}\right)_{\substack { u=0 \\
s=s \\
\begin{subarray}{c}{s=s_{10} \\
\tau_{2}=\tau_{20}{ u = 0 \\
s = s \\
\begin{subarray} { c } { s = s _ { 1 0 } \\
\tau _ { 2 } = \tau _ { 2 0 } } }\end{subarray}}=-\left(\frac{\partial^{4} p}{\partial s^{4}} \gamma^{4}\right)_{\substack{s=s_{0} \\
\tau_{1}=\tau_{10} \\
\tau_{2}=\tau_{20}}} .
$$

This can be solved to obtain

$$
\begin{aligned}
& \left(\begin{array}{l}
\frac{\partial^{4} \tau_{1}}{\partial u^{4}} \\
\frac{\partial^{4} \tau_{2}}{\partial u^{4}}
\end{array}\right)_{\substack{s=s_{0} \\
\tau_{1}=\tau_{10} \\
\tau_{2}=\tau_{20}}}= \\
& \left.-\left(\begin{array}{ll}
\operatorname{Re}\left(\frac{\partial p}{\partial \tau_{1}}\right) & \operatorname{Re}\left(\frac{\partial p}{\partial \tau_{2}}\right) \\
\operatorname{Im}\left(\frac{\partial p}{\partial \tau_{1}}\right) & \operatorname{Im}\left(\frac{\partial p}{\partial \tau_{2}}\right)
\end{array}\right)\right)_{\substack{s=s_{0} \\
\tau_{1}=\tau_{10} \\
\tau_{2}=\tau_{20}}}^{-1}\left(\begin{array}{l}
\operatorname{Re}\left(\frac{\partial^{4} p}{\partial s^{4}} \gamma^{4}\right) \\
\operatorname{Im}\left(\frac{\partial^{4} p}{\partial s^{4}} \gamma^{4}\right)
\end{array}\right) \underbrace{}_{\substack{s=s_{0} \\
\tau_{1}=\tau_{10} \\
\tau_{2}=\tau_{20}}} .
\end{aligned}
$$

Similar to the triple root case, the last equation above shows that $\left(\frac{\partial^{4} \tau_{1}}{\partial u^{4}} \frac{\partial^{4} \tau_{2}}{\partial u^{4}}\right)^{T}$ rotates four times as fast as $\gamma$ does. To understand this case, we shall divide the circle in $s$ domain in $45^{\circ}$ pieces in the complex plane, in order to work with singly connected regions (see Figures 4 to 7 , left).

Considering (12) and (13) for $\gamma=i$ and $\gamma=-i$, we see that the local stability crossing curve $\mathcal{T}_{\left(\omega_{0}, \tau_{10}, \tau_{20}\right)}$ have a cusp at $\left(\tau_{10}, \tau_{20}\right)$ [17]. Indeed, $\mathcal{T}_{\left(\omega_{0}, \tau_{10}, \tau_{20}\right)}$ partitions a sufficiently small neighborhood of $\left(\tau_{10}, \tau_{20}\right)$ into a great sector (or Gsector) and a small sector (or S-sector) as shown in Figure 1. Next theorem shows how the quadruple roots at $i \omega_{0}$ migrate as $\left(\tau_{1}, \tau_{2}\right)$ moves from $\left(\tau_{10}, \tau_{20}\right)$ to the G-sector or the $\mathbf{S}$ sector.
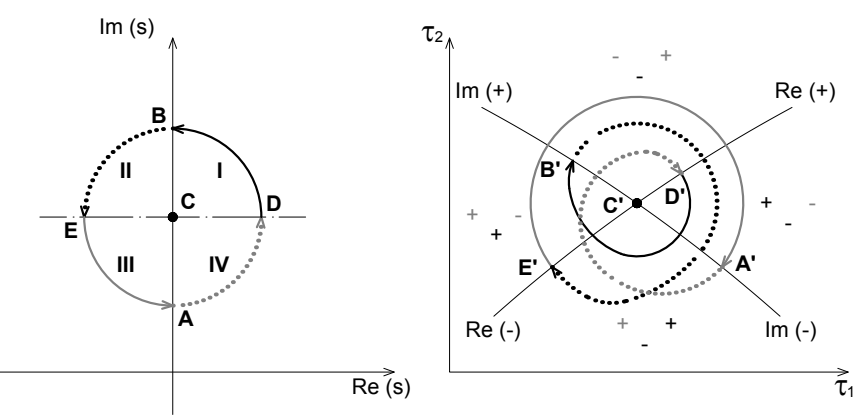

Fig. 3. The mapping $\left(\tau_{1}(s), \tau_{2}(s)\right)$ in a neighborhood of $s_{0} . D<0$.

Theorem 2: Suppose system (1) satisfies (2) and (3) for $m=4$, and assumption (4) holds.

If $\left(\tau_{1}, \tau_{2}\right)$ is in the G-sector in a sufficiently small neighborhood of $\left(\tau_{10}, \tau_{20}\right)$, then two roots of (1) in the neighborhood of $s_{0}$ are in the right half-plane, and the other two are in the left half-plane.

When $\left(\tau_{1}, \tau_{2}\right)$ is in the $\mathrm{S}$-sector, then three roots move into one half-plane, and the fourth one moves into the other half-plane. More precisely,

Case i. If $D>0$, and $\mathcal{T}_{\left(\omega_{0}, \tau_{10}, \tau_{20}\right)}^{-}$is in the counterclockwise side of $\mathcal{T}_{\left(\omega_{0}, \tau_{10}, \tau_{20}\right)}^{+}$in the S-sector, then three roots are in the left half-plane, and one root is in the right half-plane.

Case ii. If $D>0$, and $\mathcal{T}_{\left(\omega_{0}, \tau_{10}, \tau_{20}\right)}^{-}$is in the clockwise side of $\mathcal{T}_{\left(\omega_{0}, \tau_{10}, \tau_{20}\right)}^{+}$in the S-sector, then three roots are in the right half-plane, and one root is in the left half-plane.

Case iii. If $D<0$, and $\mathcal{T}_{\left(\omega_{0}, \tau_{10}, \tau_{20}\right)}^{-}$is in the counterclockwise side of $\mathcal{T}_{\left(\omega_{0}, \tau_{10}, \tau_{20}\right)}^{+}$in the S-sector, then three roots are in the right half-plane, and one root is in the left half-plane.

Case iv. If $D<0$, and $\mathcal{T}_{\left(\omega_{0}, \tau_{10}, \tau_{20}\right)}^{-}$is in the clockwise side of $\mathcal{T}_{\left(\omega_{0}, \tau_{10}, \tau_{20}\right)}^{+}$in the S-sector, then three roots are in the left half-plane, and one root is in the right half-plane.

Proof: Denote the sector $A C E$ in the left-hand side of Figures 4-7 by region $I$. In the same manner, region $I I$ the sector $E C F$, region $I I I$ the sector $F C G$, and so on. Thus, the neighbourhood of $s_{0}$ shown in left side of Figures 4 to 7 as a disk centered in $C$ is divided into 8 regions, denoted by $I, I I, \ldots, V I I I$. The mapping of these regions to the $\tau_{1}-\tau_{2}$ parameter space is represented in the right side of the figures. Note that we obtain another 8 singly connected regions: region $I^{\prime}$ is bounded by curves $A^{\prime} C^{\prime}, C^{\prime} E^{\prime}$ and $A^{\prime} E^{\prime}$, region $I I^{\prime}$ by $C^{\prime} E^{\prime}, E^{\prime} F^{\prime}$ and $F^{\prime} C^{\prime}$, and so on.

The neighbourhood $\mathcal{N}_{\varepsilon}\left(\tau_{10} \tau_{20}\right)$ is divided into S-sector and G-sector by the curves $A^{\prime} C^{\prime}$ and $B^{\prime} C^{\prime}$. In general, $F^{\prime}$ and $I^{\prime}$ each may be either in the S-sector, or in the G-sector. We shall only show the case where they are in the S-sector. Their location do not affect the validity of the conclusion. When one or both points $F^{\prime}$ and $I^{\prime}$ are outside of the Ssector, the proof for the G-sector is slightly more involved, but still possible.

Similar to the case discussed in [9] (see corollary 4) we can show that $\left(\tau_{1}(s) \tau_{2}(s)\right)$ is a bijection from $R$ to $R^{\prime}$ when $s$ is restricted to $R$, with $R$ a region from the set $\{I, I I, \ldots V I I I\}$, and $R^{\prime}$ the corresponding region in the 

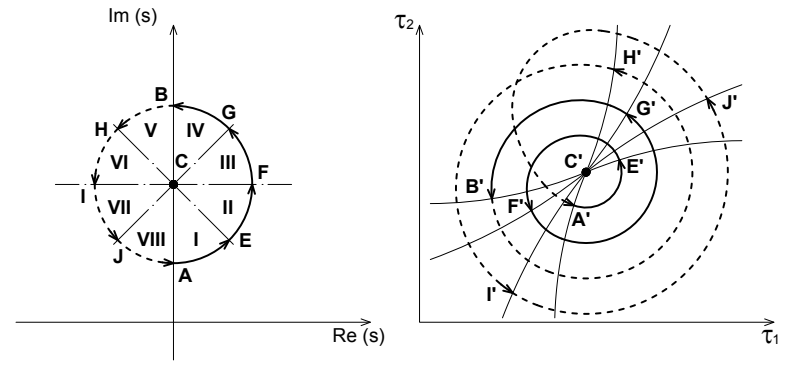

Fig. 4. The mapping $\left(\tau_{1}(s), \tau_{2}(s)\right)$ in a neighborhood of $s_{0}$. Case i: $D>0$, and $\mathcal{T}_{\left(\omega_{0}, \tau_{10}, \tau_{20}\right)}^{-}$is on the counterclockwise side of $\mathcal{T}_{\left(\omega_{0}, \tau_{10}, \tau_{20}\right)}^{+}$
in the S-sector.

set $\left\{I^{\prime}, I I^{\prime}, \ldots, V I I I^{\prime}\right\}$.

Consider Case i. The S-sector (in a sufficiently small neighbourhood) can be expressed as $\left(I I^{\prime} \cup I I I^{\prime}\right) \cap V^{\prime} \cap$ $\left(V I^{\prime} \cup V I I^{\prime}\right) \cap V I I I^{\prime}$, as depicted in Figure 4 right. But the corresponding regions are $(I I \cap I I I)$, which is in the righthalf plane, and $V,(V I \cup V I I)$ and $V I I I$, which are all in the left-half plane. So we may conclude that when $\left(\tau_{1} \tau_{2}\right)$ is in the S-sector, the characteristic equation (1) has a root in the right-half plane, and three others in the left-half plane. As for the G-sector, Figure 4 shows that it can be expressed as $\left(I^{\prime} \cup I I^{\prime}\right) \cap\left(I I I^{\prime} \cup I V^{\prime}\right) \cap\left(V^{\prime} \cup V I^{\prime}\right) \cap\left(V I I^{\prime} \cup V I I I^{\prime}\right)$. Thus, the characteristic equation (1) has two unstable roots in Gsector, within the regions $(I \cup I I)$ and $(I I I \cup I V)$, and two stable roots, within the regions $(V \cup V I)$ and $(V I I \cup V I I I)$.

Case ii: The S-sector can be expressed as $I^{\prime} \cap\left(I I^{\prime} \cup I I I^{\prime}\right) \cap$ $I V^{\prime} \cap\left(V I^{\prime} \cup V I I^{\prime}\right)$, as shown in Figure 5. Therefore, for any $\left(\tau_{1} \tau_{2}\right)$ in S-sector, one characteristic root must be in $(V I \cup V I I)$ (in the left half-plane), and the remaining three roots in right half-plane (one in $I$, one in $I I \cup I I I$, and one in $I V)$. Next, G-sector can be expressed as $\left(I^{\prime} \cup I I^{\prime}\right) \cap$ $\left(I I I^{\prime} \cup I V^{\prime}\right) \cap\left(V^{\prime} \cup V I^{\prime}\right) \cap\left(V I I^{\prime} \cup V I I I^{\prime}\right)$. Therefore, we can conclude that there are two roots on the left-half plane and two roots on the right-half plane.

For case iii and case iv, the conclusions can be drawn in a similar manner. Case iii is illustrated in Figure 6. S-sector can be expressed as $I^{\prime} \cap\left(I I^{\prime} \cup I I I^{\prime}\right) \cap I V^{\prime} \cap\left(V I^{\prime} \cup V I I^{\prime}\right)$, and G-sector as $\left(I^{\prime} \cup I I^{\prime}\right) \cap\left(I I I^{\prime} \cup I V^{\prime}\right) \cap\left(V^{\prime} \cup V I^{\prime}\right) \cap$ $\left(V I I^{\prime} \cup V I I I^{\prime}\right)$. Case iv is depicted in Figure 7, S-sector can be expressed as $\left(I I^{\prime} \cup I I I^{\prime}\right) \cap V^{\prime} \cap\left(V I^{\prime} \cup V I I^{\prime}\right) \cap V I I I^{\prime}$, and G-sector as $\left(I^{\prime} \cup I I^{\prime}\right) \cap\left(I I I^{\prime} \cup I V^{\prime}\right) \cap\left(V^{\prime} \cup V I^{\prime}\right) \cap$ $\left(V I I^{\prime} \cup V I I I^{\prime}\right)$.

\section{ILlustrative EXAMPLE}

Consider the quasi-polynomial

$$
\begin{aligned}
p\left(s, \tau_{1}, \tau_{2}\right) & =s^{4}+a_{03} s^{3}+a_{02} s^{2}+a_{01} s+a_{00}+ \\
& +\left(a_{12} s^{2}+a_{11} s+a_{10}\right) e^{-s \tau_{1}}+ \\
& +\left(a_{21} s+a_{20}\right) e^{-s \tau_{2}} .
\end{aligned}
$$

The system has a triple imaginary root at $s=s_{0}=i \omega_{0}$, with $\omega_{0}=1$, for $\left(\tau_{1} \tau_{2}\right)=(3,5), a_{03}=1, a_{12}=1$, $a_{21}=1$, and the values of other coefficients are given in table I, where $s_{k}$ stands for $\sin k$, and $c_{k}$ stands for $\cos k$.
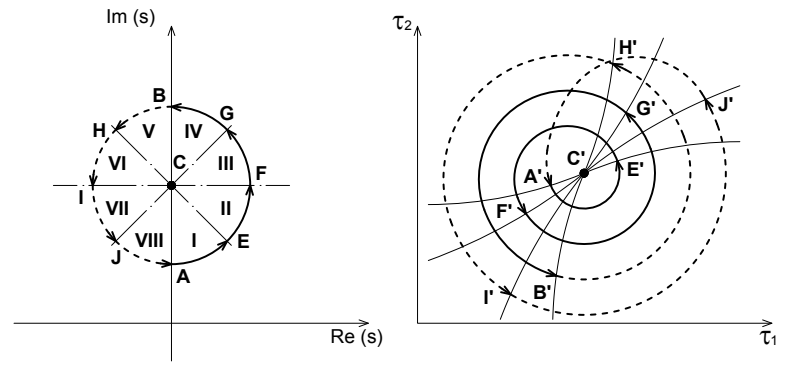

Fig. 5. The mapping $\left(\tau_{1}(s), \tau_{2}(s)\right)$ in a neighborhood of $s_{0}$. Case ii: $D>0$, and $\mathcal{T}_{\left(\omega_{0}, \tau_{10}, \tau_{20}\right)}^{-}$is on the clockwise side of $\mathcal{T}_{\left(\omega_{0}, \tau_{10}, \tau_{20}\right)}^{+}$in the S-sector.
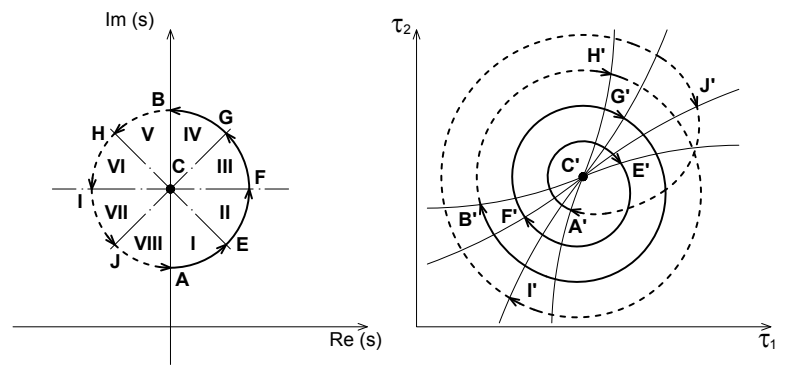

Fig. 6. The mapping $\left(\tau_{1}(s), \tau_{2}(s)\right)$ in a neighborhood of $s_{0}$. Case iii: $D<0$, and $\mathcal{T}_{\left(\omega_{0}, \tau_{10}, \tau_{20}\right)}^{-}$is on the counterclockwise side of $\mathcal{T}_{\left(\omega_{0}, \tau_{10}, \tau_{20}\right)}^{+}$ in the $\mathrm{S}$-sector.
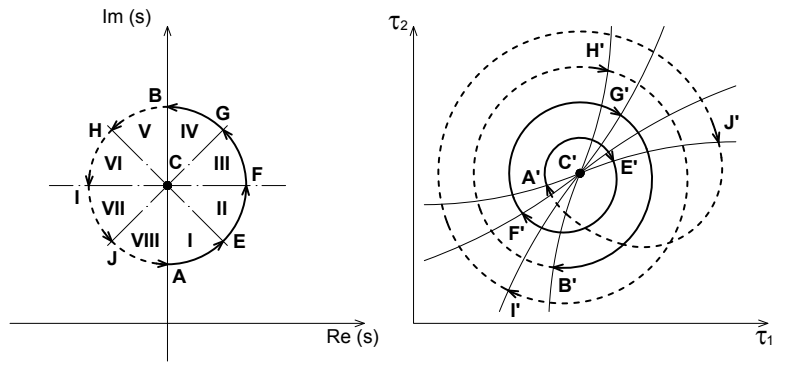

Fig. 7. The mapping $\left(\tau_{1}(s), \tau_{2}(s)\right)$ in a neighborhood of $s_{0}$. Case iv: $D<0$, and $\mathcal{T}_{\left(\omega_{0}, \tau_{10}, \tau_{20}\right)}^{-}$is on the clockwise side of $\mathcal{T}_{\left(\omega_{0}, \tau_{10}, \tau_{20}\right)}^{+}$in the S-sector.

As depicted in Figure 8, the local stability crossing curves divide the neighbourhood of $(3,5)$ in the $\tau_{1}-\tau_{2}$ plane into two regions. Next, it can be calculated that $D>0$. Therefore, for $\left(\begin{array}{lll}\tau_{1} & \tau_{2}\end{array}\right)$ taking values in the region below the curve, which is on the clockwise side of $\mathcal{T}_{\left(\omega_{0}, \tau_{10}, \tau_{20}\right)}^{-}$and on the counterclockwise side of $\mathcal{T}_{\left(\omega_{0}, \tau_{10}, \tau_{20}\right)}^{+}$, two roots will move in the left-half plane, and one root in the right-half plane. Similarly, for $\left(\begin{array}{ll}\tau_{1} & \tau_{2}\end{array}\right)$ taking values above the curve, two roots will move on the right-half plane, and one root on the left-half plane.

\section{CONCLUSIONS}

The migration of imaginary characteristic roots of multiplicity three and four in time-delay systems under the deviation of two delay parameters can be studied by using a conventional approach, without using Puiseux series.

Under the least degeneracy assumption, neither the triple 


\begin{tabular}{|c|c|}
\hline Exact value & Approx. value \\
\hline$a_{20}=\frac{630+193 s_{1}+219 s_{3}-583 s_{5}+108 s_{6}-s_{9}+2 s_{11}-172 c_{1}+216 c_{3}-10 c_{5}+18 c_{6}+2 c_{11}}{223 s_{1}-337 s_{5}+2 s_{11}+105 c_{1}+435 c_{5}}$ & $a_{02}=-2.19272$ \\
\hline$a_{11}=\frac{-58 s_{1}+1836 s_{2}+271 s_{3}-200 s_{5}+223 s_{7}+336 s_{8}-2 s_{11}+2 s_{13}+466 c_{1}-380 c_{2}-1515 c_{3}+250 c_{5}+15 c_{7}+20 c_{8}+4 c_{11}}{223 s_{1}-337 s_{5}+2 s_{11}+105 c_{1}+435 c_{5}}$ & $a_{11}=6.27284$ \\
\hline$a_{02}=\frac{-1439+606 s_{1}-418 s_{2}-5 s_{4}+1626 s_{5}-30 s_{6}+47 s_{8}+30 s_{10}+24 s_{11}-528 c_{1}+996 c_{2}-225 c_{4}+1620 c_{5}-100 c_{6}+84 c_{8}-36 c_{10}-12 c_{11}}{446 s_{1}-674 s_{5}+4 s_{11}+210 c_{1}+870 c_{5}}$ & $a_{02}=-2.19272$ \\
\hline$a_{10}=\frac{-194 s_{1}+684 s_{2}+1119 s_{3}-679 s_{5}+167 s_{7}-96 s_{8}-s_{11}-2 s_{13}+37 c_{1}-1742 c_{2}+486 c_{3}+145 c_{5}-188 c_{7}+302 c_{8}-2 c_{11}+2 c_{13}}{223 s_{1}-337 s_{5}+2 s_{11}+105 c_{1}+435 c_{5}}$ & $a_{10}=2.03748$ \\
\hline$a_{00}=\frac{-1849+1116 s_{1}-490 s_{2}-117 s_{4}+2112 s_{5}+66 s_{6}-73 s_{8}-2 s_{10}-12 s_{11}-850 c_{1}+1396 c_{2}-301 c_{4}+2430 c_{5}+76 c_{6}-38 c_{8}+44 c_{10}+4 c_{11}}{446 s_{1}-674 s_{5}+4 s_{11}+210 c_{1}+870 c_{5}}$ & $a_{00}=-2.52733$ \\
\hline$a_{01}=\frac{808+261 s_{1}+290 s_{2}+3 s_{4}-1815 s_{5}-92 s_{6}+65 s_{8}-44 s_{10}-6 s_{11}+691 c_{1}-628 c_{2}+95 c_{4}+225 c_{5}+50 c_{6}-67 c_{8}-18 c_{10}-16 c_{11}}{223 s_{1}-337 s_{5}+2 s_{11}+105 c_{1}+435 c_{5}}$ & $a_{01}=5.60094$ \\
\hline
\end{tabular}

TABLE I

COEFFICIENTS' VALUES FOR ILLUSTRATIVE EXAMPLE.

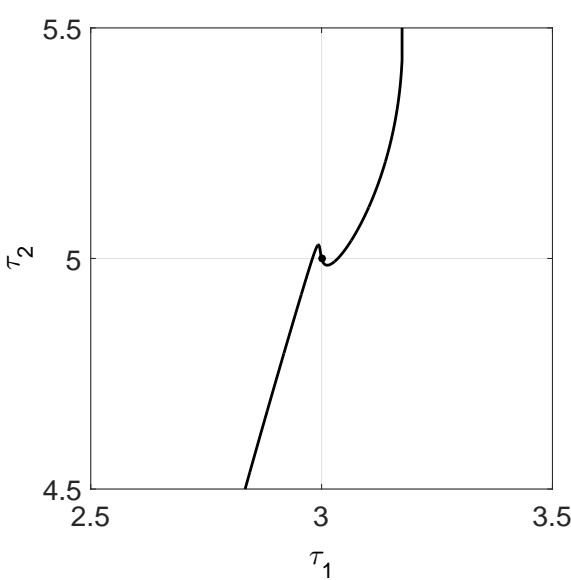

Fig. 8. The positive local stability crossing curve $\mathcal{T}_{\left(\omega_{0}, \tau_{10}, \tau_{20}\right)}^{+}$is the curve on the left-hand side of the point $\left(\tau_{10}, \tau_{20}\right)=(3,5)$. The negative local stability crossing curve $\mathcal{T}_{\left(\omega_{0}, \tau_{10}, \tau_{20}\right)}^{-}$is the curve on the right-hand side of the point $\left(\tau_{10}, \tau_{20}\right)=(3,5)$. These two curves divide $\tau_{1}-\tau_{2}$ space into two regions.

root case, nor the quadruple case may be stable under small deviation of delay parameters. More precisely, in the case of triple roots the local stability crossing curve divides the neighbourhood of $\tau_{10} \tau_{20}$ into two regions of roughly equal size. In one region, there are two roots on the right-half plane, and one root on the left-half plane. In the other region, there are two roots on the left-half plane, and one root on the right-half plane.

In the case of quadruple roots, the stability crossing curve has a cusp, and divides the neighbourhood of $\left(\tau_{10} \tau_{20}\right)$ into a S-sector and a G-sector. When the delay parameters move into the G-sector, there are two roots on the right-half plane and the other two on the left-half plane. When the delay parameters move into the S-sector, either there are three right-half plane roots and one left-half plane root, or there are three left-half plane roots and one right-half plane root.

\section{ACKNOWLEDGMENT}

We would like to thank the anonymous reviewers for their suggestions and comments. Part of the work was completed while Keqin $\mathrm{Gu}$ was visiting L2S. The financial support for this visit was provided by the RTRA Digiteo network in Paris-Saclay area.

\section{REFERENCES}

[1] E. N. Gryazina, B. T. Polyak, and A. A. Tremba, "D-decomposition technique state-of-the-art," Automation and Remote Control, vol. 69, no. 12, pp. 1991-2026, 2008.

[2] N. G. Chebotarev and N. N. Meiman, "The Routh-Hurwitz problem for polynomials and for entire functions (in Russian)," Trudy Matem. Inst. Steklov, vol. 26, pp. 1-332, 1949.

[3] L. E. El'Sgol'ts and S. B. Norkin, Introduction to the Theory and Application of Differential Equations with Deviating Arguments, Translated by J. L. Casti, Academic Press, New York, 1973.

[4] M. S. Lee and C. S. Hsu, "On the $\tau$-decomposition method of stability analysis for retarded dynamical systems," SIAM J. Control, 7:249-259, 1969.

[5] W. Michiels and S.-I. Niculescu, Stability and stabilization of timedelay systems. An eigenvalue based approach, SIAM: Philadelphia, USA, Advances in design and control, vol. 27, 2014.

[6] E. Jarlebring and W. Michiels, "Invariance properties in the root sensitivity of time-delay systems with double imaginary roots", Automatica, vol. 46, no. 6, pp. 1112-1115, 2010.

[7] X.-G. Li, S.-I. Niculescu, A. Çela, H.-H. Wang, and T.-Y. Cai, "On computing Puiseux series for multiple imaginary characteristic roots of LTI systems with commensurate delays," IEEE Trans. Autom. Control, 58, 1338-1343, 2013.

[8] K. Gu, S.-I. Niculescu, and J. Chen, "On stability of crossing curves for general systems with two delays," J. Math. Anal. Appl., vol. 311, pp. 231-253, 2005.

[9] K. Gu, D. Irofti, I. Boussaada, S.-I. Niculescu, "Migration of double imaginary characteristic roots under small deviation of two delay parameters ", IEEE 54th Conference on Decision and Control (CDC), Osaka, Japan, pp. 6410-6415, 2015.

[10] J. Sieber and B. Krauskopf, "Bifurcation analysis of an inverted pendulum with delayed feedback control near a triple-zero eigenvalue singularity ", Nonlinearity, vol. 17, nr. 1, pp. 85-103, 2004.

[11] I. Boussaada, I.-C. Morarescu, S.-I. Niculescu, "Inverted pendulum stabilization: Characterization of codimension-three triple zero bifurcation via multiple delayed proportional gains ", Systems and Control Letters, Elsevier, vol. 82, pp.1-9, 2015.

[12] T. Kato, Perturbation Theory for Linear Operators, 2nd Ed, SpringerVerlag, Berlin, 1980.

[13] K. Knopp, Theory of Functions, Parts I and II, Translated to English by F. Bagemihl, Dover, Mineola, NY, 1996.

[14] J. Chen, P. Fu, S.I. Niculescu, and Z. Guan, "An eigenvalue perturbation approach to stability analysis, part 1: eigenvalue series of matrix operators," SIAM Journal of Control Optimization, 48(8), 5564-5582, 2010.

[15] J. Chen, P. Fu, S.I. Niculescu, and Z. Guan, "An eigenvalue perturbation approach to stability analysis, part 2: when will zeros of time-delay systems cross imaginary axis?" SIAM Journal of Control Optimization, 48(8), 5583-5605, 2010.

[16] V. L. Kharitonov, S.I. Niculescu, J.A.M. Pérez and W. Michiels, "Static output feedback stabilization: necessary conditions for multiple delay controllers", IEEE Trans. Automat. Contr., 50(1), 82-86, 2005.

[17] H. W. Guggenheimer, Differential Geometry, New York: Dover, 1977. 